\title{
Definition of dispersed material layer parameters on the porous filtering partition
}

\author{
Mikhail Vasilevsky, Alexander Razva ${ }^{a}$, and Sergey Sevostyanov \\ National Research Tomsk Polytechnic University, 634050 Tomsk, Russia
}

\begin{abstract}
The simple technique for the estimation of dispersed material layer parameters on the porous partition under the gas filtration through the agglomerated particles layers is represented. The increasing of different materials density and layer porosity are analyzed.
\end{abstract}

\section{Introduction}

The filtering partitions with the different properties are applied in the apparatus for dust depletion of gases. Some filtering partitions (wears, fiber layers) are characterized by the compression under pressure drop (the thickness is decreased, the pores are deformed and decreased). The particles in gas flow are in the different conditions. The particle can goes through the pore, delays as a result of adhesion on the surface due to the particle touch, when the inertia forces act under the motion in the curving pore. The structure of deposited layer is defined by hydro-dynamic and physics-chemical factors, i.e. layer porosity, particles dimension, particles sphericity, relative humidity of gas, double electric field on the boundary dispersed material-partition, level of particles agglomeration, agglomerate deformation with the pores choking. Aggregation ability influences on the filtration process of dusting gases through the partition. During the filtration process the porous dust layer is generated on the partition, which is changed under the action of depositing particles. The average dimension and shape of the pores on the filtering partition are defined by the dimensions and shapes of partition elements and the production technology.

The allowable values of specific gas load depending on the wear kind are the following: $0.6-1.2$ for the cotton and wool wear, $0.5-1.0$ for synthetic wear, $0.5-1.0$ for glass wear.

\section{Theoretical bases}

The calculation of the sleeve wear filter leads to the definition of the total filtration surface $F_{f}$ and filters or sections number. The calculated filtration surface is defined by the formula

$$
F_{\mathrm{f}}=\frac{Q_{\mathrm{g}}+Q_{\mathrm{ba}}+Q_{\mathrm{aa}}}{v_{\mathrm{f}}} .
$$

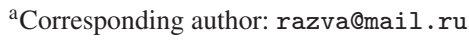

This is an Open Access article distributed under the terms of the Creative Commons Attribution License 4.0, which permits unrestricted use, distribution, and reproduction in any medium, provided the original work is properly cited. 
Here $Q_{g},\left(\mathrm{~m}^{3} / \mathrm{min}\right)$ is the rate of dusting gas, entering to the filter; $Q_{\mathrm{ba}}=(0.1-0.2) Q_{\mathrm{g}},\left(\mathrm{m}^{3} / \mathrm{min}\right)$ is the rate of blowing air (these values are less for synthetic wear); $Q_{\text {aa }},\left(\mathrm{m}^{3} / \mathrm{min}\right)$ is the rate of additional air for the temperature decrease; $v_{\mathrm{f}}$ is allowable specific load of wear filter by gas. In general the filter resistance [1] is the following:

$$
\Delta P_{\Sigma}=\mu\left(R_{0}-R_{\tau}\right) w .
$$

Here $R_{0}=r_{0} h_{0}(1 / \mathrm{m})$ is the resistance of equilibrium layer; $r_{0}=k \frac{\varphi(1-\varepsilon)^{2}}{\varepsilon^{3} \delta_{0}^{2}}\left(1 / \mathrm{m}^{2}\right)$ is specific resistance; $h_{0}=g_{0} / \rho_{\mathrm{n}},(\mathrm{m})$ is layer thickness; $g_{0}$ is equilibrium dust mass on partition pores on the surface unit; $\varphi=1 / \psi^{2}, \psi$ is shape factor; $R_{\tau}=r_{0} h_{\tau} \quad h_{\tau}=\frac{V_{\mathrm{v} \tau}}{F_{\mathrm{f}}}=\frac{C_{0} \mathcal{W} \tau}{\rho_{\tau}(1-\varepsilon)}(\mathrm{m})$ is the variable thickness of filtered dust layer; $V_{\mathrm{v} \tau},\left(\mathrm{m}^{3}\right)$ is the volume of filtered layer; $F_{\mathrm{f}},\left(\mathrm{m}^{2}\right)$ is the filter area.

However the porosity depends on the pressure and the calculating formulas must be corrected. In [1] the ways of definition of specific deposition resistance are analyzed. According to the first way the experiments on separation of researching suspension in small filter are made. The rate, the deposition volume, the pressure drop, the physical air parameters are defined. Taking into account these data they define the specific deposition resistance. According the second way the specific resistance is defined with the deposition, which was formed previously in the filter. The results coincide.

\section{Experimental setup description}

The experimental setup for the definition of the material density increasing under the filtration of the air through the dispersed material layer (Fig. 1). On Fig. 1 1.2.3 are the vessel; 4 is the researching distance; 5 is the pressure detector; 6 is the filter; 7 is the stop valve; 8 is the throttle. The filtration velocity at $\mathrm{H}$ from 50 to 100 is calculated by the formula:

$$
V_{\mathrm{f}}=\left[\frac{S h\left(P_{\mathrm{H}}+B\right)}{t\left(P_{\mathrm{d}}+B\right)}\right] / S_{\mathrm{f}} .
$$

Here $P_{\mathrm{H}},(\mathrm{kPa})$ is the additional pressure of the liquid with the height $\mathrm{H} ; P_{\mathrm{d}},(\mathrm{kPa})$ is the additional pressure of the aie in front of the measuring distance with the dispersed body; $B,(\mathrm{kPa})$ is barometric pressure; $S=344,\left(\mathrm{~cm}^{2}\right)$ is cross section area in the vessels 2 and $3 ; h=1 \mathrm{~cm}$ is the fixed change of the liquid level, when it flows from the upper vessel 2 to the lower vessel $3 . H,(\mathrm{~cm})$ is the difference of water levels in the system; $D_{\mathrm{f}}=2.50 \mathrm{~cm}$ is the filter diameter; $S_{\mathrm{f}}=4.87 \mathrm{~cm}^{2}$ is the filter area.

The experiments are made using apparatus on Fig. 2 noted on Fig. 1 as the researching distance. The apparatus is produced from the steel st 3 and consists of the operating section (1), filter (2) and additional sections (3). The diameter of the cylinder of operating section is $25 \mathrm{~mm}$, the height is $48 \mathrm{~mm}$. The pressure is registered by the manometer "Testo-512". This manometer allows one to measure the minimal and maximal pressures and the time of pressure change inside the researching distance. The material density increasing is characterized by the deviation from the linear pressure dependence on the air filtration velocity. The researching dispersed materials were the portlandcement $\mathrm{m} 400$ and abrasive micropowders "AMPEC". Granulometric composition of abrasive materials is in accordance to GOST 3647. Micropowders are the fine corundum; M40 is corundum micropowder with the particles diameter mainly $40 \mathrm{mcm}$, M14 is corundum micropowder with the particles diameter mainly $14 \mathrm{mcm}$, M5 is corundum micropowder with the particles diameter mainly $5 \mathrm{mcm}$.

\section{Experimental technique and discussion}

Micropowder or cement are propagated using the sieve with the cell about $1 \mathrm{~mm}$, then its are loaded into the main cylinder by portions (operating zone 1) (see Fig. 2), where the total mass is measured and mass the powder with small density and the density are calculated. The material after the sieve consists of agglomerated particles and has the density caused by autogesive interactions between the particles. 


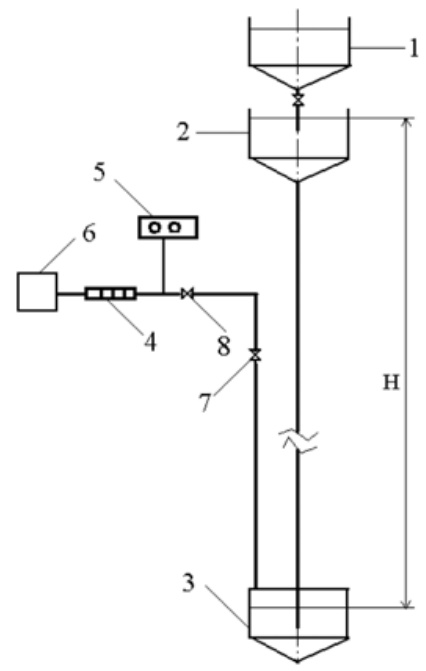

Figure 1. Experimental setup for the definition of the materials density increasing.

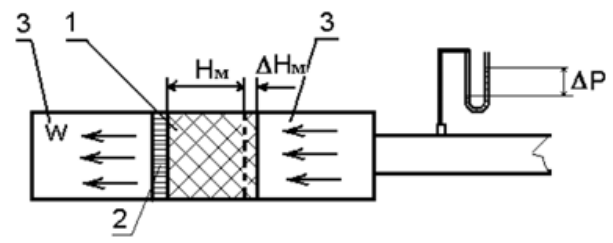

Figure 2. Apparatus for the definition of dispersed material parameters.

Cylinder volume is $V_{c}=23.5 \mathrm{~cm}^{3}, \rho_{\mathrm{m}}^{\prime}=G_{\mathrm{m}}^{\prime} / V_{\mathrm{c}}$. Cylinder mass with the filter is $G_{\mathrm{c}}=31.0 \mathrm{~g}$. The material weight inside the cylinder equals to the difference between the weight of cylinder with powder and the weight of cylinder $G_{\mathrm{m}}^{\prime}=G_{\Sigma}-G_{\mathrm{c}}$. After density increasing using filtration one associates the additional cylinder 3 (see Fig. 2), adds the powder, then removes the additional cylinder and deletes the additional powder layer from the main cylinder. Cylinders with powder are weighted to define the weight of total mass $G_{\mathrm{m}}^{\prime \prime}$ inside the cylinder. The changing of material volume after filtration is $\Delta V_{\mathrm{m}}=\frac{G_{\mathrm{m}}^{\prime \prime}-G_{\mathrm{m}}^{\prime}}{\rho_{\mathrm{m}}^{\prime}}$. The experiments were made under the slight and the sharp pressure increasing. The filtration velocity was defined by the time of water volume $\Delta Q_{\mathrm{H}}$ in the upper vessel 2 (see Fig. 1). The experimental value of $\Delta Q_{\mathrm{H}}$ equals to $344 \mathrm{~mm}^{2}$.

Every particle collides with the neighboring particles and the air, which is flowing inside pores. The pressure on the particle from the neighboring particles is the same for all dispersed layer, but the air pressure changes from layer to layer. In this case $\sigma_{z}$ is the axis strength caused by the interactions between the particles. It equals to pressure difference between the layer ends and is constant inside the layer. Under this pressure the particles shape varies and leads to decreasing of dispersed body porosity $\varepsilon$. And its resistance to air flow increases. The density ratio using this definition technique can be represented as $\rho_{\mathrm{m}}^{\prime \prime} / \rho_{\mathrm{m}}^{\prime}=1 /(1-\Delta \varepsilon)$. The estimations of the strength state of dispersed material under plunger is considered in [2]. The experimental variation of the porosity equals to $\Delta \varepsilon=\left(\rho_{\mathrm{m}}^{\prime \prime} / \rho_{\mathrm{m}}^{\prime}\right)-1$ in [2]. The comparison of these densities shows, that the density ratio in the case of plunger exceeds considerably the density ratio in the case of filtration. The contact strengths are the main, when the material density increases. The analysis shows, that the real contact strengths in material exceeds the calculated cross strengths in the case of plunger. The real contact strengths equal to pressure difference 
Table 1. Porosity variation depending on the filtration velocity.

\begin{tabular}{|c|c|c|c|c|c|}
\hline$t,(\mathbf{C})$ & $\boldsymbol{P ,}(\mathbf{k P a})$ & $V_{\mathbf{f}},(\mathbf{c m} / \mathbf{c})$ & $\rho_{\mathbf{m}}^{\prime},\left(\mathbf{g} / \mathbf{c m}^{3}\right)$ & $\left.\Delta \boldsymbol{V}_{\mathbf{m}}, \mathbf{( c m}^{3}\right)$ & $\Delta \varepsilon$ \\
\hline \multicolumn{7}{|c|}{$\mathbf{M 5}$} \\
\hline 128 & 2.5 & 0.55 & 0.74 & 2.44 & 0.104 \\
\hline 85 & 6.4 & 0.82 & 0.74 & 3.1 & 0.132 \\
\hline 51 & 9.6 & 1.38 & 0.75 & 3.73 & 0.16 \\
\hline \multicolumn{7}{|c|}{ M14 } \\
\hline 92 & 9.7 & 0.76 & 1.08 & 3.42 & 0.146 \\
\hline 98.5 & 6.7 & 0.7 & 1.1 & 2.9 & 0.124 \\
\hline 248 & 3.1 & 0.28 & 1.1 & 1.3 & 0.074 \\
\hline \multicolumn{7}{|c|}{ Cement } \\
\hline 144 & 2.2 & 0.5 & 0.89 & 2.0 & 0.085 \\
\hline 84 & 5.2 & 0.83 & 0.89 & 2.25 & 0.096 \\
\hline 38.5 & 9.8 & 1.18 & 0.89 & 3.3 & 0.13 \\
\hline \multicolumn{7}{|c|}{ M40 } \\
\hline 26 & 9.2 & 2.69 & 2.04 & 0.047 & 0.0021 \\
\hline 64 & 3.5 & 1.08 & 2.04 & 0.042 & 0.0019 \\
\hline 225 & 1.35 & 0.38 & 2.04 & 0.041 & 0.0018 \\
\hline
\end{tabular}

in the case of filtration. In this connection it is necessary to take into account the accordance between the contact strengths of plunger and filtration actions at the same pressures.

The results of experiment are presented in the Table 1 . The density of material layer under the filtration was calculated by the formula: $\rho_{\mathrm{m}}^{\prime}=G_{\mathrm{m}}^{\prime} / V_{\mathrm{c}}$. The porosity variation is calculated by the formula: $\Delta \varepsilon=\Delta V_{\mathrm{m}} / V_{\mathrm{c}}$.

The density variation of the material M40 equals to $9.2 \%$ (see Table 1). Consequently, the dispersed layer with the medium grain dimension does not change the density under the filtration. The specific resistance depends on the porosity and specific area surface of the solid particles. In the process of gas filtration through the porous partition the deposited dust is in the agglomerated state. The layer density increasing under the particles accumulation is connected with the reformation of aggregations.

There are the different relations for the connection of the porosity and the particles dimension. In [1] the technique for calculation of specific deposition resistance $r_{0} /(1-\varepsilon)$ is represented. The particle diameter is $r_{0} /(1-\varepsilon)=2300 / \delta^{2}$. In the connection of the considerable increasing of specific deposition resistance with the increasing of the small particles concentration one can use the relation $r_{0} /(1-\varepsilon)=r_{\mathrm{cm}}, r_{\mathrm{cm}}=\left(r_{1} / r_{2}\right)^{M_{\mathrm{v}} /\left[0.85\left(\delta_{2} / \delta_{1}\right)^{2}+0.5\right]}$, Here $r_{1}$ and $r_{2}$ are the specific deposition resistances for the particles with the diameters $\delta_{1}$ and $\delta_{2}$ accordingly; $M_{\mathrm{v}}$ is the part of the fine particles in the mix.

In [3] the data for increasing of the material with the high density is presented. The density change at the pressure equals to $100 \mathrm{kPa}$ is $6.5 \%$. If one considers the gas motion as the linear through the pores system, one can use Gagen-Puaseile equation $W=S_{\mathrm{k}} \frac{\delta_{\mathrm{e}}^{2} \Delta P}{32 \mu h_{\mathrm{r} 1}}$. Here $S_{\mathrm{k}}$ is the total area of effective pores cross-section devided by the unit of filter area; $\delta_{\mathrm{e}}$ is the equivalent particles diameter; $S_{k} \approx \varepsilon, \delta_{\mathrm{e}}=\frac{4 \varepsilon}{S_{0}(1-\varepsilon)}$, where $S_{0}$ is the specific surface area of deposition solid particles. Coseni-Karmann equation [3] shows, that $S_{0}=\sqrt{\frac{\varepsilon^{2}}{k(1-\varepsilon)} \times \frac{\Delta P}{\mu h_{\mathrm{r} 1} W}}$.

\section{Conclusions}

The data obtained on proposed technique for the estimation of dispersed material layer parameters on the porous partition show, that one can use given technique to forecast the dust density increasing on wear partitions of the industrial filters. This technique is enough simple and chip. 
Thermophysical Basis of Energy Technologies

\section{References}

[1] V. Zhuzhikov. Filtration: Theory and practice of suspensions separation. (Chimia, Moscow, 1980).

[2] M. Vasilevsky, K. Nekrasova, A.Razva, E. Zykov. Zavodskaya laboratoria, 75, 32 (2009)

[3] V. Voronin, V. Egorov. The problems of air hydrodynamics and heat mass currency, 85 (TSU, Tomsk, 1983) 